Article

\title{
The Transcriptome of Brassica napus L. Roots under Waterlogging at the Seedling Stage
}

\section{Xiling Zou, Xiaoyu Tan, Chengwei Hu, Liu Zeng, Guangyuan Lu, Guiping Fu, Yong Cheng and Xuekun Zhang *}

Key Laboratory of Biology and Genetic Improvement of Oil Crops, Ministry of Agriculture, Oil Crops Research Institute of the Chinese Academy of Agricultural Sciences, Wuhan 430062, China; E-Mails: zouxiling@gmail.com (X.Z.); tanxy85@163.com (X.T.); chw63@126.com (C.H.); zeng88liu@163.com (L.Z.); luwiz@oilcrops.cn (G.L.); fgp928076@hotmail.com (G.F.); chengyong58@yahoo.com.cn (Y.C.)

* Author to whom correspondence should be addressed; E-Mail: seedcq@263.net; Tel./Fax: +86-27-8682-4573.

Received: 27 November 2012; in revised form: 11 January 2013 / Accepted: 14 January 2013 / Published: 28 January 2013

\begin{abstract}
Although rapeseed (Brassica napus L.) is known to be affected by waterlogging, the genetic basis of waterlogging tolerance by rapeseed is largely unknown. In this study, the transcriptome under $0 \mathrm{~h}$ and $12 \mathrm{~h}$ of waterlogging was assayed in the roots of ZS9, a tolerant variety, using digital gene expression (DGE). A total of 4432 differentially expressed genes were identified, indicating that the response to waterlogging in rapeseed is complicated. The assignments of the annotated genes based on GO (Gene Ontology) revealed there were more genes induced under waterlogging in "oxidation reduction", "secondary metabolism", "transcription regulation", and "translation regulation"; suggesting these four pathways are enhanced under waterlogging. Analysis of the 200 most highly expressed genes illustrated that 144 under normal conditions were down-regulated by waterlogging, while up to 191 under waterlogging were those induced in response to stress. The expression of genes involved under waterlogging is mediated by multiple levels of transcriptional, post-transcriptional, translational and post-translational regulation, including phosphorylation and protein degradation; in particular, protein degradation might be involved in the negative regulation in response to this stress. Our results provide new insight into the response to waterlogging and will help to identify important candidate genes.
\end{abstract}


Keywords: rapeseed (Brassica napus L.); waterlogging; DGE (digital gene expression); roots; transcriptome

\section{Introduction}

Waterlogging is one of the most widespread abiotic determinants for crop growth, leading to the depletion of oxygen, which is vital to plants [1]. The depletion of oxygen is a major feature of waterlogging because the diffusion of oxygen in water is $10^{-4}$ times slower than that in air [2]. The imbalance between the slow diffusion of gases and the rate that oxygen is consumed by micro-organisms and plant roots drastically reduces the supply of oxygen [3], which is vital to the roots of plant.

During recent years, gene expression studies in Arabidopsis [4-7], maize [8,9], rice [10,11], and other species [12-14] exposed to low oxygen have demonstrated that low oxygen stress causes drastic changes in gene expression. Although the expression of a majority of global genes was depressed, the accumulation of mRNAs was revealed for many genes under hypoxia. These genes included anaerobic proteins (ANPs) involved in sugar phosphate metabolism [15]. Studies have subsequently identified signal transduction components that are involved in the activation of RopGAP4 (Rop GTPase activating protein4) [2,16], and transient induction of mitochondrial alternative oxidase (AOX), induction of calmodulin and CAP (calmodulin-associated peptide) [15,17,18]. Moreover, the induction of plant growth regulators under waterlogging stress are involved in signaling cascades that influence cellular responses, including increases in ethylene [19,20], abscisic acid (ABA) [21-25], gibberellic acid (GA) [26], and auxin (IAA) [27,28] and a reduction in cytokinin (CK) [29,30]. Transcriptional factors (TFs) play an extremely important role in waterlogging tolerance. In rice, two TFs, Snorkel [31] and Submergence-1A [32], have been cloned by mapped based cloning, and both of them encode ethylene-responsive factor-type transcription factors that have evolved opposite functions to adapt to different types of flood. In Arabidopsis, studies have revealed that oxygen sensing is mediated by group VII ERF (ethylene response factor) TFs through the N-End rule pathway [33].

Although many transcriptomic studies on waterlogging have addressed similar topics with regard to gene expression in response to waterlogging, this response has proven to have a very complex mechanism. Indeed, understanding the mechanisms that coordinate the regulation of waterlogging tolerance remains a fundamental challenge. Furthermore, there is still no report of a large-scale of gene expression analysis of the response to waterlogging in rapeseed (Brassica napus L.).

Rapeseed is particularly sensitive to waterlogging. The plants experience waterlogging when directly sown in paddy field planted as a rotation crop following rice in China, the largest rapeseed-planting country in the world [29,30]. Because there is a need to understand the response to waterlogging in rapeseed, it is necessary and helpful to study expression profiles under waterlogging in a tolerant variety of rapeseed.

To gain comprehensive insight into how rapeseed responds to waterlogging and to identify the genes important in mounting a response of waterlogging tolerance, here we report a detailed analysis of gene expression profiling in ZS9, a waterlogging-tolerant variety [29,30], at the vegetative growth stage under waterlogging using digital gene expression (DGE) method, a powerful tool for studying high-throughput gene expression profiling [34,35]. We identified sets of positively and negatively 
significantly expressed genes in response to waterlogging. Our analysis suggests that waterlogging affects a broad spectrum of functional categories and that the regulation of waterlogging tolerance is complex, involving with multiple levels of regulation. The mechanism of the response to waterlogging is discussed.

\section{Results and Discussion}

\subsection{Results}

\subsubsection{Analysis of DGE Libraries}

To identify genes in response to waterlogging, RNA libraries were generated using the roots of ZS9 seedlings at $0 \mathrm{~h}$ (the control) and $12 \mathrm{~h}$ after waterlogging (the treatment) (Table 1). More than 30 million original sequencing tags were produced, representing 13,457,553 and 19,621,584 raw reads from the library of $0 \mathrm{~h}$ and $12 \mathrm{~h}$, respectively. The junk tags were filtered (low quality tags, tags with one copy, tags containing $\mathrm{N}$ and tags of low quality) prior to mapping these tag sequences to the reference sequences, producing approximately 13.3 and 19.4 million clean sequence tags, respectively. For the two libraries, $57.32 \%$ and $60.31 \%$ of the clean tags were mapped unambiguously, with $4,221,453(31.78 \%$ of the clean tags) and $6,684,936(34.52 \%$ of the clean tags) clean tags being perfectly mapped with a stringent criterion of 0 mismatches within the 16-nucleotide tag alignments. Some $3.94 \%$ and $4.71 \%$ of the clean tags mapped to duplicated genes, alternate transcripts, or repeated sequences. Lastly, 30,964 and 28,954 unique genes representing 7,614,486 and 11,679,339 DGE tags from the control and the treatment libraries were obtained, and the counts for each unique gene were normalized to the reads per kb per million reads (RPKM) for the two libraries.

Table 1. Summary of the two DGE (digital gene expression) libraries.

\begin{tabular}{|c|c|c|}
\hline & $\mathbf{O} \mathbf{h}$ & $12 \mathrm{~h}$ \\
\hline Raw Reads & $13,457,553$ & $19,621,584$ \\
\hline Clean Reads & $13,283,443$ & $19,364,949$ \\
\hline \multirow[t]{2}{*}{ Mapped Reads } & $8,138,298$ & $12,591,493$ \\
\hline & $61.27 \%(8,138,298 / 13,283,443)$ & $65.02 \%(12,591,493 / 19,364,949)$ \\
\hline \multirow[t]{2}{*}{ Perfect match } & $4,221,453$ & $6,684,936$ \\
\hline & $51.87 \%{ }^{b}$ & $53.09 \%{ }^{b}$ \\
\hline \multirow[t]{2}{*}{$\leq 2$ bp mismatch } & $3,916,845$ & $5,906,557$ \\
\hline & $29.49 \%^{a} \quad 48.13 \%^{b}$ & $30.5 \%^{\mathrm{a}} \quad 46.91 \%^{\mathrm{b}}$ \\
\hline \multirow[t]{2}{*}{ Unique match } & $7,614,486$ & $11,679,339$ \\
\hline & $93.56 \%{ }^{b}$ & $60.31 \%^{a} \quad 92.76 \%^{b}$ \\
\hline \multirow{2}{*}{ Multi-position match } & 523,812 & 912,154 \\
\hline & $6.44 \%$ b & $4.71 \%^{\mathrm{a}}$ \\
\hline Unmapped Reads & $\begin{array}{l}5,145,145 \\
38.73 \%{ }^{\mathrm{a}} \\
\end{array}$ & $\begin{array}{l}6,773,456 \\
34.98 \%{ }^{a} \\
\end{array}$ \\
\hline
\end{tabular}

Additionally, the sequencing saturation was analyzed in the two libraries to estimate whether the sequencing depth was sufficient for the transcriptomic coverage. The genes mapped by all clean tags and unambiguous clean tags increased with the total number of tags. When the sequencing counts reached 2.5 million tags or higher, the number of detected genes was saturated (Figure S1), indicating that the sequencing depth was sufficient for both of the two libraries. ${ }^{\text {a }}$ was represented the ratio was from the comparison between the number of sequences and the number of clean reads; ${ }^{\mathbf{b}}$ was represented the ratio was from the comparison between the number of sequences and the number of mapped reads. 


\subsubsection{Changes in Global Gene Transcription under Waterlogging}

To characterize the genes involved in the response to waterlogging in roots at the seedling stage, the expression profiles at $12 \mathrm{~h}$ (the treatment) and $0 \mathrm{~h}$ (the control) of waterlogging were compared. A statistical analysis of the frequency of genes identified 4432 differentially expressed genes under waterlogging (Supplementary Data 1).

Figure 1. Functional categorization of all the annotated differentially expressed genes. This analysis was based on 2387 annotated genes, not including genes with "unknown function" or "no homology".

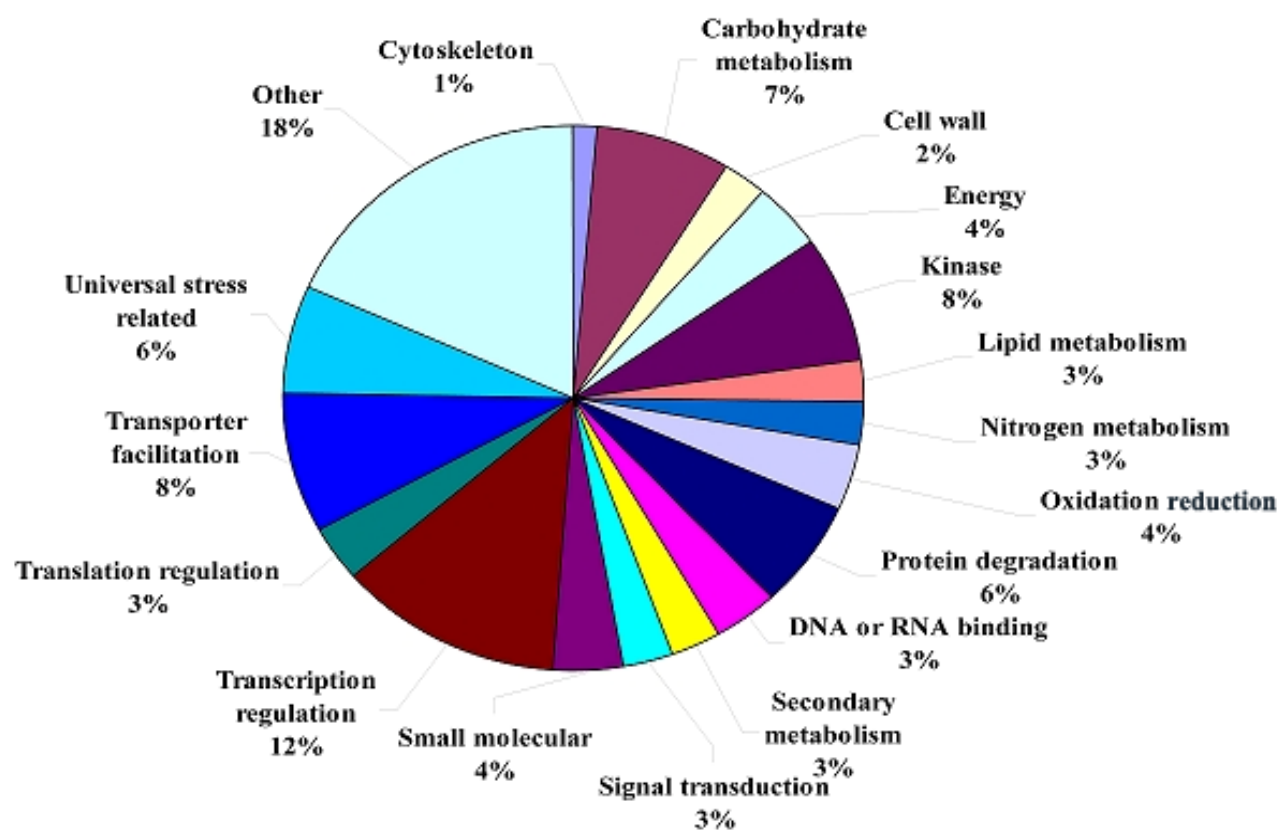

An annotation analysis revealed that nearly half $(45.6 \%, 2019 / 4432)$ of the differentially expressed genes were "functional unknown", annotated as "unnamed protein product", "hypothetical protein" or "unknown protein". Moreover, 26 genes, accounting for $0.6 \%$ of all the differentially expressed genes, did not match to known sequences, defined as "no homology", which suggested our study may allow for the identification of novel genes in the response to waterlogging tolerance. Based on GO, 2387 annotated genes were categorized into 18 functional categories (Figure 1). The largest categories were "transcription regulation" (12.4\%), "transporter facilitation" (8.3\%), and "kinase" (7.6\%). The expected group associated with "carbohydrate metabolism" represented for 178 genes. In addition to "carbohydrate metabolism", genes related to "lipid metabolism" (61 genes) and "nitrogen metabolism" (60 genes) were also identified, representing for $2.6 \%$ and $2.5 \%$, respectively. As we expected, 151 genes $(6.5 \%)$ were found to be categorized into the group of "universal stress related". We also noticed a high percentage of genes related to "protein degradation" (152 genes). Interestingly, 83 genes (3.5\%) involved in "DNA or RNA binding" were found to be differentially expressed under waterlogging. Additionally, the categories related to "energy" and "small molecular" each contained 92 genes (3.9\%. A total of 93 genes (3.9\%) involved in "oxidation reduction" were found. In addition to the regulation of transcription in response to waterlogging revealed by the largest group of genes related to "transcription regulation", a set of 82 (3.4\%) differentially expressed genes were involved in 
"translation regulation". Substantial sets also included the groups of "signal transduction" (3.0\%) and "secondary metabolism" (2.7\%). 35 genes involved in "cytoskeleton" and 58 genes related to "cell wall" were also identified. A lot of significant genes (18.4\%) from a wide variety of pathways were affected by waterlogging and were categorized into the group of "other function".

\subsubsection{Transcriptomic Comparison of the Roots under Waterlogging Treatment and the Control Using} DGE Tag Profiling

Of the 4432 differentially expressed genes, 1709 genes were up-regulated and 2723 genes were down-regulated. Based on the categorization of up-regulated and down-regulated genes respectively, we performed a comparison between these categories. Since there were more genes down-regulated under waterlogging compared that up-regulated as expected, there were more down-regulated genes than up-regulated ones in most categories (Figure 2). However, there were more genes up-regulated in response to waterlogging in the four categories of "oxidation reduction", "secondary metabolism", "transcription regulation", and "translation regulation". Additionally, only six genes with no homology were identified as being down-regulated under waterlogging, whereas there were up to 20 novel genes up-regulated in response to this stress. To provide further valuable information, gene Ontology (GO) assignments were performed based on the annotated genes up-regulated and down-regulated genes, respectively. As shown in Figure 3, the top three largest groups of up-regulated genes were "transcription regulation", "transporter facilitation", and "universal stress related" under normal conditions, while under waterlogging, "transcription regulation", "kinase", and "carbohydrate metabolism" were the top three largest groups. Surprisingly, genes related to "transcription regulation" comprised up to $18.6 \%$ of the up-regulated genes.

Figure 2. Comparison between up-regulated and down-regulated genes based on function categories.

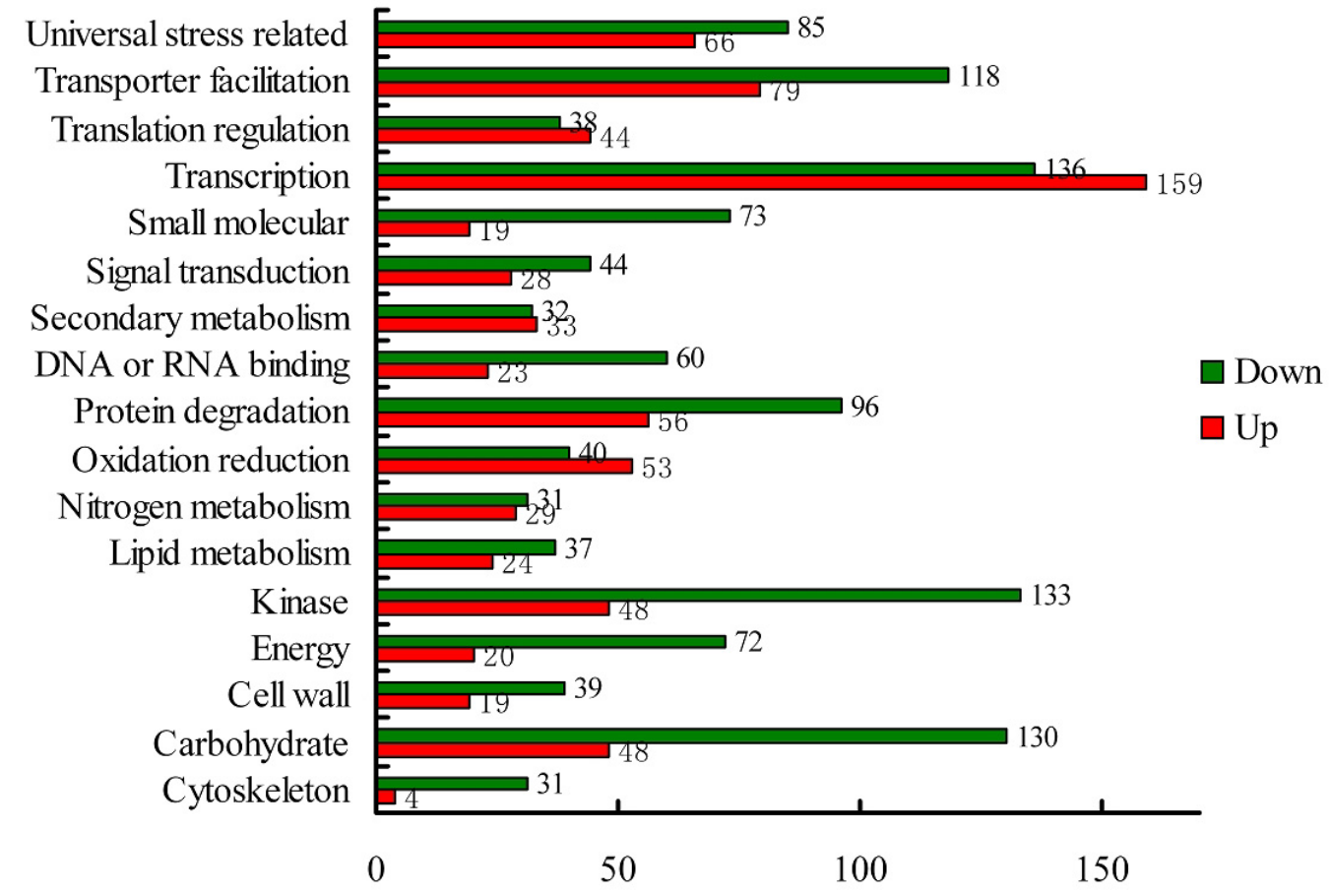


Figure 3. Functional categorization of up-regulated and down-regulated expressed genes. This analysis did not include the genes with "no function annotation" or "no homology".

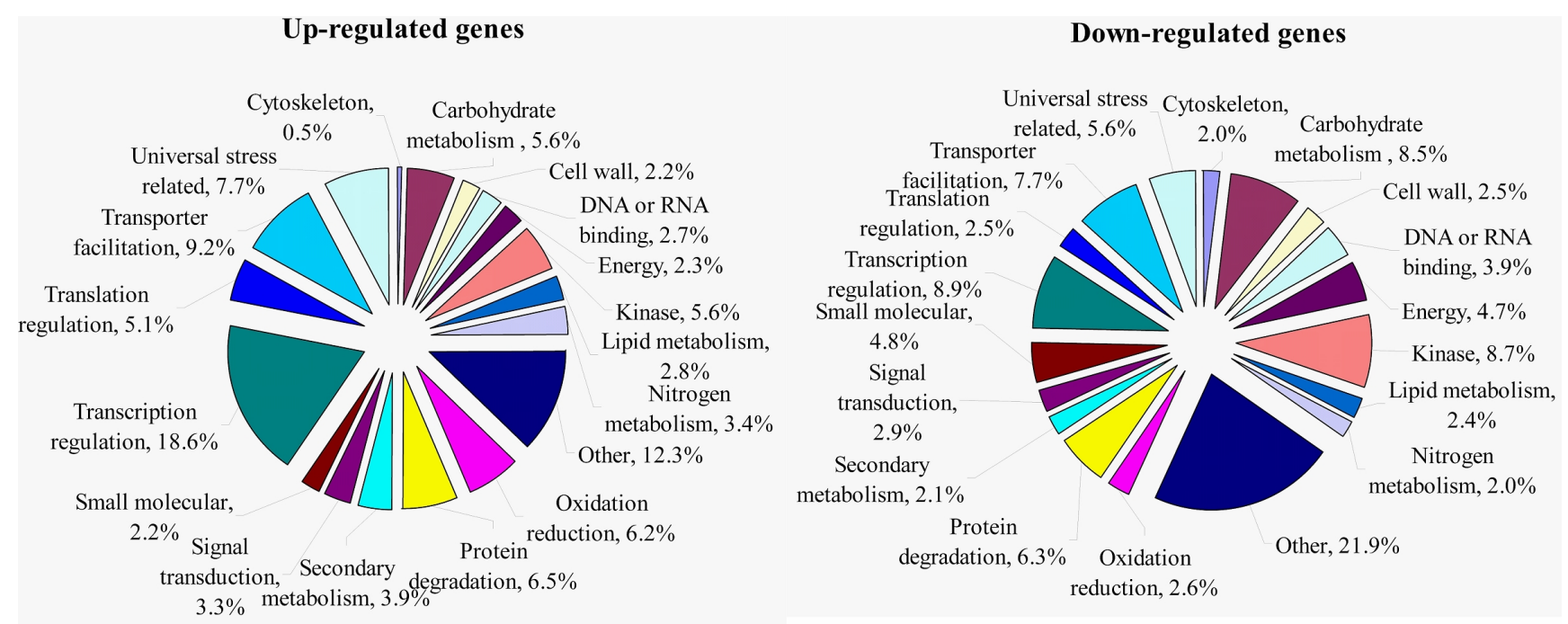

2.1.4. Expression Levels of Differentially Expressed Genes under the Control and Waterlogging Stress

High-throughput sequencing can provide information about gene expression levels, and we observed that the majority of transcripts were represented by a few genes with abundant counts. Considering the abundance of all the differentially expressed genes under the control and the stress, the transcriptome at $0 \mathrm{~h}$ (the control) and $12 \mathrm{~h}$ (the treatment) under waterlogging condition consist of unevenly distributed sequence abundance in which the top 200 unique genes with the highest expression level accounted for $14.7 \%$ and $32.6 \%$ of the total counts of transcripts, respectively (Supplementary Data 2). The 200 most highly expressed genes in both samples were grouped based on their function (Table 2). 144 out of the top 200 abundant genes under the normal condition were down-regulated when waterlogging occurred, while under waterlogging up to 191 ones were those genes induced in response to the stress. Although most of the highly expressed genes under the control were down-regulated in response to the stress, the pathways of "universal stress related", "carbohydrate metabolism", "translation regulation", and "energy" were enriched under waterlogging.

\subsubsection{Verification of The DGE Data by Real-Time Quantitative RT-PCR}

To validate the results of the DGE data, the transcriptional level of 12 unigenes were examined by real time PCR. Although the change fold did not exactly match the number revealed by the DGE data for these genes, all the unigenes showed consistent expression patterns that were consisted with the DGE data, and exhibited $>2$ fold higher expression in response to waterlogging (Table 3). The ANP encoding genes, including glyceraldehyde-3-phosphate dehydrogenase and alcohol dehydrogenase, were chosen in the real time PCR. The results showed that these two genes were up-regulated significantly under waterlogging indicated by both DGE data and verified by real time PCR, indicating that our experimental results for conditions of waterlogging were valid. 
Table 2. Distribution of the top 200 highly expressed genes under normal and waterlogging condition.

\begin{tabular}{|c|c|c|c|c|c|c|c|c|c|c|c|}
\hline Categories & $\begin{array}{c}\text { Control } \\
\text {-total }\end{array}$ & $\%^{a}$ & $\begin{array}{c}\text { Control } \\
\text {-down }\end{array}$ & $\%^{b}$ & $\begin{array}{c}\text { Control } \\
\text {-up }\end{array}$ & $\%^{c}$ & $\begin{array}{c}\text { Waterlogged } \\
\text {-total }\end{array}$ & $\%^{d}$ & $\begin{array}{c}\text { Waterlogged } \\
\text {-up }\end{array}$ & $\%^{\mathrm{e}}$ & $\begin{array}{c}\text { Waterlogged } \\
\text {-down }\end{array}$ \\
\hline Unknown function & 79 & $39.5 \%$ & 60 & $41.7 \%$ & 19 & $33.9 \%$ & 84 & $42.0 \%$ & 80 & $41.9 \%$ & 4 \\
\hline Universal stress related & 17 & $8.5 \%$ & 10 & $6.9 \%$ & 7 & $12.5 \%$ & 20 & $10.0 \%$ & 18 & $9.4 \%$ & 2 \\
\hline Carbohydrate metabolism & 13 & $6.5 \%$ & 8 & $5.6 \%$ & 5 & $8.9 \%$ & 12 & $6.0 \%$ & 12 & $6.3 \%$ & 0 \\
\hline Transporter facilitation & 10 & $5.0 \%$ & 7 & $4.9 \%$ & 3 & $5.4 \%$ & 11 & $5.5 \%$ & 10 & $5.2 \%$ & 1 \\
\hline Other & 15 & $7.5 \%$ & 12 & $8.3 \%$ & 3 & $5.4 \%$ & 9 & $4.5 \%$ & 9 & $4.7 \%$ & 0 \\
\hline Translation regulation & 8 & $4.0 \%$ & 2 & $1.4 \%$ & 6 & $10.7 \%$ & 8 & $4.0 \%$ & 8 & $4.2 \%$ & 0 \\
\hline Protein degradation & 8 & $4.0 \%$ & 5 & $3.5 \%$ & 3 & $5.4 \%$ & 8 & $4.0 \%$ & 8 & $4.2 \%$ & 0 \\
\hline Oxidation reduction & 6 & $3.0 \%$ & 4 & $2.8 \%$ & 2 & $3.6 \%$ & 7 & $3.5 \%$ & 7 & $3.7 \%$ & 0 \\
\hline No homology & 4 & $2.0 \%$ & 1 & $0.7 \%$ & 3 & $5.4 \%$ & 7 & $3.5 \%$ & 6 & $3.1 \%$ & 1 \\
\hline Transcription regulation & 4 & $2.0 \%$ & 3 & $2.1 \%$ & 1 & $1.8 \%$ & 6 & $3.0 \%$ & 6 & $3.1 \%$ & 0 \\
\hline Nitrogen metabolism & 5 & $2.5 \%$ & 4 & $2.8 \%$ & 1 & $1.8 \%$ & 6 & $3.0 \%$ & 6 & $3.1 \%$ & 0 \\
\hline Lipid metabolism & 3 & $1.5 \%$ & 2 & $1.4 \%$ & 1 & $1.8 \%$ & 5 & $2.5 \%$ & 5 & $2.6 \%$ & 0 \\
\hline Kinase & 3 & $1.5 \%$ & 3 & $2.1 \%$ & 0 & $0.0 \%$ & 4 & $2.0 \%$ & 4 & $2.1 \%$ & 0 \\
\hline Signal transduction & 5 & $2.5 \%$ & 5 & $3.5 \%$ & 0 & $0.0 \%$ & 3 & $1.5 \%$ & 2 & $1.0 \%$ & 1 \\
\hline Secondary metabolism & 5 & $2.5 \%$ & 4 & $2.8 \%$ & 1 & $1.8 \%$ & 3 & $1.5 \%$ & 3 & $1.6 \%$ & 0 \\
\hline Energy & 3 & $1.5 \%$ & 2 & $1.4 \%$ & 1 & $1.8 \%$ & 2 & $1.0 \%$ & 2 & $1.0 \%$ & 0 \\
\hline DNA or RNA binding & 0 & $0.0 \%$ & 0 & $0.0 \%$ & 0 & $0.0 \%$ & 2 & $1.0 \%$ & 2 & $1.0 \%$ & 0 \\
\hline Cell wall & 5 & $2.5 \%$ & 5 & $3.5 \%$ & 0 & $0.0 \%$ & 2 & $1.0 \%$ & 2 & $1.0 \%$ & 0 \\
\hline Small molecular & 2 & $1.0 \%$ & 2 & $1.4 \%$ & 0 & $0.0 \%$ & 1 & $0.5 \%$ & 1 & $0.5 \%$ & 0 \\
\hline Cytoskeleton & 5 & $2.5 \%$ & 5 & $3.5 \%$ & 0 & $0.0 \%$ & 0 & $0.0 \%$ & 0 & $0.0 \%$ & 0 \\
\hline Total & 200 & - & 144 & - & 56 & - & 200 & - & 191 & - & 9 \\
\hline Percentage $^{\mathrm{f}}$ & - & - & $72.0 \%$ & & $28.0 \%$ & & - & - & $95.5 \%$ & & $4.5 \%$ \\
\hline
\end{tabular}

${ }^{\mathbf{a}}$ and ${ }^{\mathbf{d}}$ represent for the percentage of genes accounting of the top 200 highly expressed genes under normal and waterlogging condition, respectively; ${ }^{\mathbf{b}}$ represents for the percentage of genes accounting of induced ones under normal condition, while ${ }^{\mathbf{c}}$ represents for reduced ones; ${ }^{\mathbf{e}}$ represents the percentage of genes accounting of induced ones under waterlogging; ${ }^{\mathbf{f}}$ represents the percentage of induced and reduced genes under normal and waterlogging condition. 
Table 3. Verification of DGE results by real time PCR.

\begin{tabular}{|c|c|c|c|}
\hline Gene ID & Annotation & Fold change by DGE ${ }^{a}$ & Fold change by Q-PCR \\
\hline Bra038700 & polygalacturonase inhibitory protein & 3.2 & $9.8 \pm 1.4$ \\
\hline Bra021558 & nine-cis-epoxycarotenoid dioxygenase 3 & 3.1 & $4.9 \pm 1.1$ \\
\hline Bra003701 & $A P 2$ domain containing protein $R A P 2.5$ & 2.5 & $7.4 \pm 1.5$ \\
\hline Bra014080 & hydrolase & 2.4 & $24.9 \pm 1.1$ \\
\hline Bra007609 & glycoside hydrolase family 28 protein & 2.3 & $12.2 \pm 1.3$ \\
\hline Bra016729 & glyceraldehyde-3-phosphate dehydrogenase 1 & 4.1 & $6.4 \pm 1.3$ \\
\hline Bra022115 & transcription factor & 2.1 & $8.9 \pm 1.2$ \\
\hline Bra004778 & Stearoyl-acyl carrier protein desaturase & 17.0 & $73.0 \pm 1.4$ \\
\hline $\operatorname{Bra} 012551$ & abscisic acid 8'-hydroxylase/ oxygen binding & 0.3 & $0.24 \pm 0.01$ \\
\hline Bra019528 & betaine aldehyde dehydrogenase & 0.3 & $0.3 \pm 0.01$ \\
\hline Bra015693 & alcohol dehydrogenase & 16.2 & $12.1 \pm 1.2$ \\
\hline Bra030945 & phosphoenolpyruvate carboxylase & 0.3 & $0.2 \pm 0.03$ \\
\hline
\end{tabular}

The transcriptional level of candidate genes was examined by real time PCR with three biological replications of RNA and actin was used as an internal control.

\subsection{Discussion}

The technology of DGE by high-throughput sequencing is now in common in use for transcriptomic analyses. In this study, we applied this method to evaluate gene expression under waterlogging in the roots of seedlings for rapeseed. As expected, many genes were differentially expressed when waterlogging occurred; some of these genes were further confirmed by real-time PCR experiments, demonstrating the validity of the DGE data. The GO analysis revealed that these differentially expressed genes were distributed among various pathways, suggesting that a wide spectrum of physiological processes was affected by the low-oxygen stress.

Although a relatively large number of genes were down-regulated by waterlogging, the pathways associated with "secondary metabolism", "oxidation reduction", "transcription regulation", and "translation regulation" were enhanced under waterlogging, which confirms the current knowledge of the waterlogging response. It is apparent that a battery of signaling molecules, such as ethylene, ABA, GA, IAA and reduction of $\mathrm{CK}$, is modulated during stressful conditions, and it is not surprising that the pathway of "secondary metabolism" was enhanced under waterlogging [21-30]. Moreover, ROS (reactive oxygen species) production has been suggested to be a component of signaling under hypoxia [36-38]. Genes responsible for ROS handling, such as cytochrome c oxidase, peroxidase, and NADH-ubiquinone oxidoreductase, etc., were identified in our study, which is consistent with the previous report that the induction of oxidative metabolism is necessary for the induction of adaptive responses to waterlogging [36,39-41]. The pathways of "transcription regulation" and "translation regulation" were enhanced under the imposed stress, suggesting regulation of gene expression at both the transcriptional and translational levels, as discussed below.

\subsubsection{Protein Degradation May Be Involved in Negative Regulation in the Response to Waterlogging}

Under normal conditions, $0 \mathrm{~h}$ (the control) in our study, the genes for development (called "developmental genes" herein) in various pathways (Table 2) were among the 200 most abundant genes and were distributed almost all categories. However, these "developmental genes" were 
down-regulated upon waterlogging. In our study, 2387 genes were reduced in response to waterlogging. The ratio of the number of reduced genes compared to induced genes was more than 1.5. Additionally, the expression level decreased for $72 \%$ of the 200 most highly expressed "developmental genes", with only 10 of these genes remaining in the list of the 200 most highly expressed genes under waterlogging. This result is accordance with similar studies in Arabidopsis, rice, maize and other species. It is a widely accepted fact that the genes encoding enzymes for development under normal conditions, such as phosphoenolpyruvate carboxylase, pyrophosphate-fructose-6-phosphate 1-phosphotransferase and glucose-6-phosphate dehydrogenase (related to aerobic respiration) [9,39], which are not necessary for survival under waterlogging stress, are down-regulated when waterlogging occurs.

When a plant grows under normal conditions, different proteins encoded by different genes are expressed at different development stages, and transcription of the genes related to the previous stage should be down-regulated when the plant enters the next development stage, and the existing proteins should be degraded because they are no longer required. As showed in our study, 4\% of the 200 most highly expressed genes at $0 \mathrm{~h}$ (the control) (Table 2) were genes involved in protein degradation and should play an important role in degrading the proteins encoded by "developmental genes" under normal conditions. As mentioned by Zou [8], the degradation of aerobic proteins would help decrease the consumption of oxygen and supply free amino acids for breakdown of carbon skeletons for the supply of energy under waterlogging. In our study, 56 genes were up-regulated in response to waterlogging (Figure 2) and may be involved in the above pathways.

Recently, a breakthrough in the study on waterlogging tolerance revealed that the N-end rule pathway of protein degradation acts as a homeostatic sensor of severely low oxygen levels in Arabidopsis through the regulation of key hypoxia-response TFs [33,42,43]. To some extent, the mechanism in Arabidopsis is similar to that in animals, in which the proteasomal degradation of key transcription factor hypoxia-induced factors (HIFs) depending on the proline hydroxylation controls the global response to hypoxia [44]. In other words, protein degradation plays a negative role in response to hypoxia. In our study, 96 genes were identified as being down-regulated by the stress, accounting for up to $6.3 \%$ of the reduced genes, some of which might be involved in the negative regulation of important protein in the response to waterlogging. In most transcriptomic studies under waterlogging, the up-regulated genes have more often been highlighted than the down-regulated genes based on the fact that hypoxia represses the expression of many genes. Indeed, very little is known about the role of gene repression in waterlogging tolerance. Our analysis includes the potential function of genes involved in protein degradation that were down-regulated, providing a new insight for future studies.

\subsubsection{Multiple Levels of Regulation Were Involved in the Response to Waterlogging}

Plants respond to waterlogging in various ways.

Firstly, transcriptional regulation clearly plays an important role in response to waterlogging. Expression of 4432 genes was altered significantly under waterlogging in this study. Therefore, it is not surprising that the category of "transcription regulation" showed the largest number of differentially expressed genes, up to $12.4 \%$. TFs have attracted considerable interest in previous studies. Some key genes regulating waterlogging that have been cloned in plants are TF encoding genes, including Sub1A, Snorkel1, Snorkel2, HRE1, HRE2, and RAP2.2 [33,42,43,45], and, in 
particular, ethylene response factor (ERF). In the present study, seven genes coding ERFs were identified as being regulated, and five were induced: including ERF2, ERF4, ERF7, ERF11, and ERF54. The importance of these genes will require further verification.

Secondly, some reports have demonstrated that miRNA and alternative splicing play important regulation roles in response to waterlogging [46-48]. To support this, among the induced genes identified under waterlogging in this study, there was a category containing a considerable number of genes related to "DNA/RNA binding" (Figure 1), such as RNA recognition motif (RRM)-containing protein, $R N A$-binding protein-like protein, and $R N A$-binding protein cp 31 , which might be involved in the post-transcriptional regulation that occurs with this stress.

Thirdly, a variability in the efficiency of protein synthesis for different genes under waterlogging have been reported before [49] and is based on differing abilities of the transcripts of different genes to associate with the translational complexes [39]. A total of 82 genes involved in "translation regulation" were differentially expressed in response to waterlogging in this study. With global decrease in protein synthesis, it is interesting to find that 44 genes related to translation were up-regulated, including glutamyl-tRNA synthetase, translation initiation factor, and ribosomal protein L17 family protein. Considering of the information above, a question remains whether there are different translational machineries serving for the transcripts of different genes, leading to different protein synthesis efficiencies. Under waterlogging, some translation machinery might regulate the synthesis efficiency of different proteins depending on the quantity of the translation complexes themselves, in addition to the regulation of transcript abundance.

Finally, the pathway of phosphorylation plays an important role in signal transduction through regulating phosphorylation of specific protein [8]. We found that the expression level of genes related to phosphorylation, including purple acid phosphatase 17, tyrosine specific protein phosphatase family protein, were altered under waterlogging, These genes might regulate the activity of target proteins though the regulation of their own abundance. Additionally, the protein degradation discussed above is also involved in the regulation of gene activity under waterlogging.

\section{Experimental Section}

\subsection{Plant Materials and Waterlogging Treatment}

ZS9 [29,30] with high waterlogging tolerance was used in this study. Seeds were germinated on moist filter paper. After three days, germinated seeds were individually transplanted to sand chambers. All the plants were grown with $16 / 8 \mathrm{~h}$ day/night cycles at $30{ }^{\circ} \mathrm{C} / 22{ }^{\circ} \mathrm{C}$ and a light intensity of $500 \mu \cdot \mathrm{mol} \cdot \mathrm{m}^{-2} \cdot \mathrm{s}^{-1}$. Seedlings with two leaves were used. Uniform seedlings were selected and divided into two groups: one group was cultured with normal water supply as the control and the other was submerged in water with all leaves in air as the treatment (Figure S2). Roots treated for $12 \mathrm{~h}$ and roots of the controls were harvested at the same time, and were stored at $-80{ }^{\circ} \mathrm{C}$.

\subsection{RNA Isolation}

Total RNA was isolated using TRIzol (Invitrogen, California, CA, USA) according to the manufacture's instructions followed by RNase-free DNase treatment (Takara, Dalian, China). RNA quantity and quality were assessed by a Nanodrop spectrophotometer and by agarose gel electrophoresis. 


\subsection{DGE-Tag Profiling}

Two DGE libraries were constructed using total RNA of roots of seedlings waterlogged for $12 \mathrm{~h}$ and that of the control with Illumina's Digital Gene Expression Tag Profiling Kit according to the manufacturer's protocol (Version 2.1B). The two tag libraries underwent Illumina proprietary sequencing chip for cluster generation through in situ amplification and were deep-sequenced using Illumina Genome Analyzer. The image files generated were processed to produce digital-quality sequence data.

For the raw data, low quality tags, adaptor sequences, tags with unknown nucleotides $\mathrm{N}$, empty reads, tags that were too short or too long, and tags with only one copy, were filtered to get clean reads. The types of clean tags were represented as the distinct clean tags. Subsequently, we classified the clean tags and distinct clean tags according to their copy number in the library and showed their percentage in the total clean and distinct tags, and analyzed saturation of the two libraries.

For annotation, all the tags were mapped to the reference sequences, including NCBI EST database of Brassica napus L., and unigenes of the Brassica oleracea Genomics Database because there is no genome sequence of Brassica napus L. and its genome (AC genome) is a polyploidy of Brassica rapa genome (A genome) and Brassica oleracea genome (C genome). Only no more than 1-bp nucleotide mismatch was allowed.

\subsection{Identification of Differentially Expressed Genes}

The expression level of each gene was normalized to RPKM based on the number of clean tags. Genes were deemed significantly differentially expressed with a $p$-value $<0.005$, FDR $<0.01$ and a relative change threshold of two-fold in the sequence counts across libraries. Functional classification of differentially expressed genes was carried out according to the functional categories of GO.

\subsection{Quantitative Real-Time PCR Analysis}

Three biological replications with two technique replications of total RNA were used for quantitative real-time PCR analysis. Total RNA was treated with RNase-free DNase. Reverse transcription of total RNA (5 $\mu$ g) was performed with M-MLV RTase cDNA Synthesis Kit (Takara, Dalian, China).

Real time PCR was carried out using a CFX96 Real-Time System C1000 Thermal Cycler (Bio-RAD, Hercules, CA, USA) using SYBRGreen PCR Master Mix (Takara, Dalian, China). Primers were designed using PRIMER3 software [50] and were listed in Table S1. The expression of actin was used as a control. PCR amplification conditions and the data analysis were referred to Zou's report [8].

\section{Conclusions}

In summary, based on several pieces of evidence derived from this study, we speculate that the response to waterlogging is mediated by the regulation of the levels of transcription, post-transcription, translation and post-translation, including phosphorylation and protein degradation. Certainly, it is better to reveal the changes in the real activity of genes under waterlogging through metabolite profiling [13,51-53], which can consider all the levels of regulation. However, because the study of 
genes transcription is more convenient and less consuming and expensive, this approach is still a good choice to study the abundance of transcripts from the beginning of regulation, which also affects other levels of regulation.

A major objective of our study was to reveal the mechanism of waterlogging tolerance. Our study has demonstrated that gene regulation in response to oxygen deprivation is mediated by transcription, post-transcription, translation, and post-translation, including phosphorylation and protein degradation; in particular, protein degradation might be involved in the negative regulation of the stress response. A large number of differentially expressed genes always leads to difficulty in the characterization of the genes that are actually related to waterlogging tolerance. Based on our analysis, the genes related to multiple levels of regulation might be good choice for further study. This analysis provides a good starting point for future functional studies.

\section{Acknowledgments}

This work is supported by the Hi-Tech Research and Development program of China (2011AA10A104), National Science Foundation of China (31171589 and 31201244), the National Basic Research Program (2011CB109302) and Special Fund for Agroscientific Research in the Public Interest (2101203032).

\section{Conflict of Interest}

The authors declare no conflict of interest.

\section{References}

1. Subbaiah, C.C.; Sachs, M.M. Molecular and cellular adaptations of maize to flooding stress. Ann. Bot. 2003, 91, 119-127.

2. Dat, J.F.; Capelli, N.; Folzer, H.; Bourgeade, P.; Badot, P.M. Sensing and signalling during plant flooding. Plant Physiol. Biochem. 2004, 42, 273-282.

3. Jackson, M.B.; Colmer, T.D. Response and adaptation by plants to flooding stress. Ann. Bot. 2005, 96, 501-505.

4. Liu, F.; Vantoai, T.; Moy, L.P.; Bock, G.; Linford, L.D.; Quackenbush, J. Global transcription profiling reveals comprehensive insights into hypoxic response in Arabidopsis. Plant Physiol. 2005, 137, 1115-1129.

5. Gonzali, S.; Loreti, E.; Novi, G.; Poggi, A.; Alpi, A.; Perata, P. The use of microarrays to study the anaerobic response in Arabidopsis. Ann. Bot. 2005, 96, 661-668.

6. Hsu, F.C.; Chou, M.Y.; Peng, H.P.; Chou, S.J.; Shih, M.C. Insights into hypoxic systemic responses based on analyses of transcriptional regulation in Arabidopsis. PLoS One 2011, 6, e28888.

7. Van Dongen, J.T.; Frohlich, A.; Ramirez-Aguilar, S.J.; Schauer, N.; Fernie, A.R.; Erban, A.; Kopka, J.; Clark, J.; Langer, A.; Geigenberger, P. Transcript and metabolite profiling of the adaptive response to mild decreases in oxygen concentration in the roots of arabidopsis plants. Ann. Bot. 2009, 103, 269-280. 
8. Zou, X.; Jiang, Y.; Liu, L.; Zhang, Z.; Zheng, Y. Identification of transcriptome induced in roots of maize seedlings at the late stage of waterlogging. BMC Plant Biol. 2010, 10, 189.

9. Peschke, V.M.; Sachs, M.M. Characterization and expression of transcripts induced by oxygen deprivation in maize (Zea mays L.). Plant Physiol. 1994, 104, 387-394.

10. Agarwal, S.; Grover, A. Isolation and transcription profiling of low- $\mathrm{O}_{2}$ stress-associated cDNA clones from the flooding-stress-tolerant FR13A rice genotype. Ann. Bot. 2005, 96, 831-844.

11. Lasanthi-Kudahettige, R.; Magneschi, L.; Loreti, E.; Gonzali, S.; Licausi, F.; Novi, G.; Beretta, O.; Vitulli, F.; Alpi, A.; Perata, P. Transcript profiling of the anoxic rice coleoptile. Plant Physiol. 2007, 144, 218-231.

12. Christianson, J.A.; Llewellyn, D.J.; Dennis, E.S.; Wilson, I.W. Global gene expression responses to waterlogging in roots and leaves of cotton (Gossypium hirsutum L.). Plant Cell Physiol. 2010, $51,21-37$.

13. Nanjo, Y.; Maruyama, K.; Yasue, H.; Yamaguchi-Shinozaki, K.; Shinozaki, K.; Komatsu, S. Transcriptional responses to flooding stress in roots including hypocotyl of soybean seedlings. Plant Mol. Biol. 2011, 77, 129-144.

14. Qi, X.H.; Xu, X.W.; Lin, X.J.; Zhang, W.J.; Chen, X.H. Identification of differentially expressed genes in cucumber (Cucumis sativus L.) root under waterlogging stress by digital gene expression profile. Genomics 2012, 99, 160-168.

15. Subbaiah, C.C.; Kollipara, K.P.; Sachs, M.M. A Ca ${ }^{2+}$-dependent cysteine protease is associated with anoxia-induced root tip death in maize. J. Exp. Bot. 2000, 51, 721-730.

16. Baxter-Burrell, A.; Yang, Z.; Springer, P.S.; Bailey-Serres, J. RopGAP4-dependent Rop GTPase rheostat control of Arabidopsis oxygen deprivation tolerance. Science 2002, 296, 2026-2028.

17. Bailey-Serres, J.; Chang, R. Sensing and signalling in response to oxygen deprivation in plants and other organisms. Ann. Bot. 2005, 96, 507-518.

18. Manjunath, S.; Williams, A.J.; Bailey-Serres, J. Oxygen deprivation stimulates $\mathrm{Ca}^{2+}$-mediated phosphorylation of mRNA cap-binding protein eIF4E in maize roots. Plant J. 1999, 19, 21-30.

19. Barnawal, D.; Bharti, N.; Maji, D.; Chanotiya, C.S.; Kalra, A. 1-Aminocyclopropane-1-carboxylic acid (ACC) deaminase-containing rhizobacteria protect Ocimum sanctum plants during waterlogging stress via reduced ethylene generation. Plant Physiol. Biochem. 2012, 58, 227-235.

20. Hess, N.; Klode, M.; Anders, M.; Sauter, M. The hypoxia responsive transcription factor genes ERF71/HRE2 and ERF73/HRE1 of Arabidopsis are differentially regulated by ethylene. Physiol. Plant 2011, 143, 41-49.

21. Yang, S.H.; Choi, D. Characterization of genes encoding ABA 8'-hydroxylase in ethylene-induced stem growth of deepwater rice (Oryza sativa L.). Biochem. Biophys. Res. Commun. 2006, 350, 685-690.

22. De Bruxelles, G.L.; Peacock, W.J.; Dennis, E.S.; Dolferus, R. Abscisic acid induces the alcohol dehydrogenase gene in Arabidopsis. Plant Physiol. 1996, 111, 381-391.

23. Else, M.A.; Davies, W.J.; Malone, M.; Jackson, M.B. A negative hydraulic message from oxygen-deficient roots of tomato plants? (influence of soil flooding on leaf water potential, leaf expansion, and synchrony between stomatal conductance and root hydraulic conductivity). Plant Physiol. 1995, 109, 1017-1024. 
24. Neuman, D.S.; Smit, B.A. The influence of leaf water status and aba on leaf growth and stomata of phaseolus seedlings with hypoxic roots. J. Exp. Bot. 1991, 42, 1499-1506.

25. Dennis, E.S.; Dolferus, R.; Ellis, M.; Rahman, M.; Wu, Y.; Hoeren, F.U.; Grover, A.; Ismond, K.P.; Good, A.G.; Peacock, W.J. Molecular strategies for improving waterlogging tolerance in plants. J. Exp. Bot. 2000, 51, 89-97.

26. He, C.J.; Morgan, P.W.; Drew, M.C. Transduction of an ethylene signal is required for cell death and lysis in the root cortex of maize during aerenchyma formation induced by hypoxia. Plant Physiol. 1996, 112, 463-472.

27. Klok, E.J.; Wilson, I.W.; Wilson, D.; Chapman, S.C.; Peacock, W.J.; Dolferus, R.; Dennis, E.S. Expression profile analysis of the low-oxygen response in Arabidopsis root cultures. Plant Cell 2002, 14, 2481-2494.

28. Visser, E.; Cohen, J.D.; Barendse, G.; Blom, C.; Voesenek, L. An ethylene-mediated increase in sensitivity to auxin induces adventitious root formation in flooded Rumex palustris Sm. J. Exp. Bot. 1996, 112, 1687-1692.

29. Chen, J.; Zhang, X.; Chen, L.; Chao, G.; Li, J. Evaluation of waterlogging tolerance germplasm in rapeseed (Brassica napus L.) with germinated seeds with anoxic stress. Chin. J. Oil Crop Sci. 2006, 28, 138-143.

30. Cheng, Y.; Gu, M.; Cong, Y.; Zou, C.; Zhang, X.; Wang, H. Combining ability and genetic effects of germination traits of Brassica napus L. under waterlogging stress condition. Agric. Sci. China 2010, 9, 101-105.

31. Hattori, Y.; Nagai, K.; Furukawa, S.; Song, X.J.; Kawano, R.; Sakakibara, H.; Wu, J.; Matsumoto, T.; Yoshimura, A.; Kitano, H. The ethylene response factors SNORKEL1 and SNORKEL2 allow rice to adapt to deep water. Nature 2009, 460, 1026-1030.

32. Xu, K.; Xu, X.; Fukao, T.; Canlas, P.; Maghirang-Rodriguez, R.; Heuer, S.; Ismail, A.M.; Bailey-Serres, J.; Ronald, P.C.; Mackill, D.J. Sub1A is an ethylene-response-factor-like gene that confers submergence tolerance to rice. Nature 2006, 442, 705-708.

33. Sasidharan, R.; Mustroph, A. Plant oxygen sensing is mediated by the N-end rule pathway: A milestone in plant anaerobiosis. Plant Cell 2011, 23, 4173-4183.

34. Linsen, S.E.; Cuppen, E. Methods for small RNA preparation for digital gene expression profiling by next-generation sequencing. Methods Mol. Biol. 2012, 822, 205-217.

35. Hong, L.Z.; Li, J.; Schmidt-Kuntzel, A.; Warren, W.C.; Barsh, G.S. Digital gene expression for non-model organisms. Genome Res. 2011, 21, 1905-1915.

36. Pucciariello, C.; Parlanti, S.; Banti, V.; Novi, G.; Perata, P. Reactive oxygen species-driven transcription in Arabidopsis under oxygen deprivation. Plant Physiol. 2012, 159, 184-196.

37. Banti, V.; Mafessoni, F.; Loreti, E.; Alpi, A.; Perata, P. The heat-inducible transcription factor HsfA2 enhances anoxia tolerance in Arabidopsis. Plant Physiol. 2010, 152, 1471-1483.

38. Chang, R.; Jang, C.J.; Branco-Price, C.; Nghiem, P.; Bailey-Serres, J. Transient MPK6 activation in response to oxygen deprivation and reoxygenation is mediated by mitochondria and aids seedling survival in Arabidopsis. Plant Mol. Biol. 2012, 78, 109-122.

39. Branco-Price, C.; Kawaguchi, R.; Ferreira, R.B.; Bailey-Serres, J. Genome-wide analysis of transcript abundance and translation in Arabidopsis seedlings subjected to oxygen deprivation. Ann. Bot. 2005, 96, 647-660. 
40. Ellis, M.H.; Dennis, E.S.; Peacock, W.J. Arabidopsis roots and shoots have different mechanisms for hypoxic stress tolerance. Plant Physiol. 1999, 119, 57-64.

41. Keller, T.; Damude, H.G.; Werner, D.; Doerner, P.; Dixon, R.A.; Lamb, C. A plant homolog of the neutrophil NADPH oxidase gp91phox subunit gene encodes a plasma membrane protein with $\mathrm{Ca}^{2+}$ binding motifs. Plant Cell 1998, 10, 255-266.

42. Gibbs, D.J.; Lee, S.C.; Isa, N.M.; Gramuglia, S.; Fukao, T.; Bassel, G.W.; Correia, C.S.; Corbineau, F.; Theodoulou, F.L.; Bailey-Serres, J. Homeostatic response to hypoxia is regulated by the N-end rule pathway in plants. Nature 2011, 479, 415-418.

43. Licausi, F.; Kosmacz, M.; Weits, D.A.; Giuntoli, B.; Giorgi, F.M.; Voesenek, L.A.C.J.; Perata, P.; van Dongen, J.T. Oxygen sensing in plants is mediated by an N-end rule pathway for protein destabilization. Nature 2011, 479, 419-422.

44. Ivan, M.; Kondo, K.; Yang, H.; Kim, W.; Valiando, J.; Ohh, M.; Salic, M.; Asara, J.M.; Lane, W.S.; Kaelin, W.G., Jr. HIFalpha targeted for VHL-mediated destruction by proline hydroxylation: Implications for $\mathrm{O}_{2}$ sensing. Science 2001, 292, 464-468.

45. Hinz, M.; Wilson, I.W.; Yang, J.; Buerstenbinder, K.; Liewellyn, D.; Dennis, E.S.; Sauter, M.; Dolferus, R. Arabidopsis RAP2.2: An ethylene response transcription factor that is important for hypoxia survival. Plant Physiol. 2010, 153, 757-772.

46. Zhai, L.; Liu, Z.; Zou, X.; Jiang, Y.; Qiu, F.; Zheng, Y.; Zhang, Z. Genome-wide identification and analysis of microRNA responding to long-term waterlogging in crown roots of maize seedlings. Physiol. Plant 2012, 147, 181-193.

47. Zou, X.; Jiang, Y.; Zheng, Y.; Zhang, M.; Zhang, Z. Prolyl 4-hydroxylase genes are subjected to alternative splicing in roots of maize seedlings under waterlogging. Ann. Bot. 2011, 108, 1323-1335.

48. Moldovan, D.; Spriggs, A.; Yang, J.; Pogson, B.J.; Dennis, E.S.; Wilson, I.W. Hypoxia-responsive microRNAs and trans-acting small interfering RNAs in Arabidopsis. J. Exp. Bot. 2010, 61, 165-177.

49. Chang, W.W.; Huang, L.; Shen, M.; Webster, C.; Burlingame, A.L.; Roberts, J.K.M. Patterns of protein synthesis and tolerance of anoxia in root tips of maize seedlings acclimated to a low-oxygen environment, and identification of proteins by mass spectrometry. Plant Physiol. 2000, 122, 295-318.

50. Ye, J.; Coulouris, G.; Zaretskaya, I.; Cutcutache, I.; Rozen, S.; Madden, T.L. Primer-BLAST: A tool to design target-specific primers for polymerase chain reaction. BMC Bioinforma. 2012, 13, 134.

51. Libourel, I.G.; van Bodegom, P.M.; Fricker, M.D.; Ratcliffe, R.G. Nitrite reduces cytoplasmic acidosis under anoxia. Plant Physiol. 2006, 142, 1710-1717.

52. Dixon, M.H.; Hill, S.A.; Jackson, M.B.; Ratcliffe, R.G.; Sweetlove, L.J. Physiological and metabolic adaptations of Potamogeton pectinatus L. tubers support rapid elongation of stem tissue in the absence of oxygen. Plant Cell Physiol. 2006, 47, 128-140.

53. Ratcliffe, R.G. In vivo NMR studies of the metabolic response of plant tissues to anoxia. Ann. Bot. 1997, 79, 39-48.

(C) 2013 by the authors; licensee MDPI, Basel, Switzerland. This article is an open access article distributed under the terms and conditions of the Creative Commons Attribution license (http://creativecommons.org/licenses/by/3.0/). 